\title{
Discussions informelles au sein du groupe de travail et construction du stress
}

Le cas des infirmières hospitalières et des policiers de sécurité urbaine

\section{Marc Loriol}

\section{OpenEdition \\ Journals}

Édition électronique

URL : http://journals.openedition.org/communicationorganisation/877

DOI : 10.4000/communicationorganisation.877

ISSN : 1775-3546

\section{Éditeur}

Presses universitaires de Bordeaux

\section{Édition imprimée}

Date de publication : 1 décembre 2009

Pagination : 20-31

ISBN : 978-2-86781-719-9

ISSN : $1168-5549$

\section{Référence électronique}

Marc Loriol, « Discussions informelles au sein du groupe de travail et construction du stress », Communication et organisation [En ligne], 36 | 2009, mis en ligne le 19 avril 2011, consulté le 19 avril 2019. URL : http://journals.openedition.org/communicationorganisation/877 ; DOI : 10.4000/ communicationorganisation.877 
Dossier : Pour une approche communicationnelle de l'individu au travail

\title{
Résumé
}

À partir d'une étude sur la manière dont policiers et infirmières parlent de leur stress au travail, cet article aborde la façon dont le sens du travail et finalement les difficultés et le stress sont construits collectivement à travers les échanges informels au cours de l'activité. En donnant une vision positive des tâches effectuées, en posant les limites du «beau travail » et de l'engagement auprès des usagers, les discussions quotidiennes permettent plus ou moins de gérer symboliquement les problèmes et d'éviter qu'ils ne débouchent sur des plaintes de stress.

\section{Mots clefs}

Policiers, infirmières, construction sociale, stress, échanges informels, collectifs de travail.

\begin{abstract}
From a study on the way police officers and nurses speak about their stress at work, this article tackles how the meaning of work difficulties and stress are collectively constructed through informal exchange during activity. By forging a positive view of the task realised and putting bonds to the "nice work" and the involvement toward users problems, the day-to-day talks help more or less to manage troubles and avoid then to become stress complains.
\end{abstract}

\section{Keywords}

Police officers, nurses, social construction, stress, Informal exchanges, work collectives.

Marc Loriol est chargé de recherche au CNRS au laboratoire Georges Friedmann (Université de Paris 1). Il a travaillé sur la construction sociale des risques psychosociaux au travail et les métiers des services publics. 


\section{Discussions informelles au sein du groupe de travail et construction du stress. Le cas des infirmières hospitalières et des policiers de sécurité urbaine Marc Loriol}

marc.loriol@orange.fr

Il est fréquent, dans les entretiens menés avec des salariés à propos de leurs conditions de travail, d'entendre les personnes interrogées déclarer que les aspects pénibles de leurs activités sont compensés par une «bonne ambiance» (soutien des collègues, échange de plaisanteries, camaraderie...). De leur côté, les spécialistes du stress ont souligné, depuis le travail fondateur de Sydney Cobb (1976), l'importance de ce qu'ils appellent le «soutien social», défini comme toute « information qui conduit le sujet à croire que l'on prend soin de lui, qu'il est aimé, estimé, qu'il fait partie d'un réseau d'obligations mutuelles »(Cobb, 1976 : 300). L'objet de cet article est de montrer comment les communications dans le travail construisent collectivement le sens des situations et des difficultés rencontrées (Foster, Bochner, 2008). Les échanges informels et langagiers dans et sur le travail n'ont pas seulement pour effet d'apporter des informations et du réconfort, mais contribuent au cadrage de l'activité, à la catégorisation des usagers à la définition même des réalités affrontées.

L'intérêt de comparer (Vigour, 2004) deux groupes professionnels dont le travail est réputé stressant (comme le montrent les nombreuses publications sur le stress des policiers et des infirmières ${ }^{9}$ ou les classifications du Bureau International du Travail) est de mettre en évidence des modes spécifiques de production des représentations du travail et du stress. Une étude collective ${ }^{10}$, menée dans quatre

${ }^{9}$ Pour une présentation de quelques unes, cf. Loriol, Boussard et Caroly (2004).

${ }^{10}$ Menée avec Valérie Boussard et Sandrine Caroly entre 2003 et 2005, cette étude a commencé par une phase d'observation (de deux à trois mois par établissement) des patrouilles de police et des équipes soignantes, suivie, quelques mois plus tard d'entretiens semi-directifs (133) avec une partie des agents observés. Il s'agissait de comprendre comment étaient catégorisées et perçues les contraintes au travail, la façon dont elles étaient « psychologisées » ou traitées comme des questions d'organisation du travail. Les discussions spontanées dans les voitures de police ou en salle de soins, le vocabulaire pour décrire les situations et les usagers ont été particulièrement 
Dossier : Pour une approche communicationnelle de l'individu au travail

commissariats de police et trois hôpitaux sur la façon dont les difficultés relationnelles dans le travail étaient perçues et gérées, a montré l'importance des échanges sur le travail entre collègues pour faire face aux situations pénibles. Le fait de parler de ses problèmes à autrui est bénéfique, non pas par un effet de catharsis ou d'abréaction, comme le pensait Freud (1909), mais parce que cela est l'occasion de construire collectivement un sens acceptable pour les événements à l'origine des émotions négatives (Rimé, 2005).

\section{Échanger pour cadrer le travail}

Travailler, notamment dans les métiers relationnels, c'est maîtriser son environnement, contrôler concrètement ou au moins symboliquement les usagers avec qui les salariés sont en contact (Hugues, 1997; Milburn, 2002). Par les échanges quotidiens d'expériences, d'anecdotes, de plaisanteries, les salariés vont forger, de façon plus ou moins solide suivant les cas, une version ou une représentation particulière de la situation, ce que Goffman (1974) appelle, à la suite de Bateson (1977), un cadre. Ces cadres sont faits de routines, de savoirs pratiques, de procédures collectives de travail, de division implicite des tâches, de valeurs partagées.

Le travail de police-secours offre un bon terrain d'observation pour les communications professionnelles. Le temps des policiers peut difficilement être rationalisé car leur activité est dépendante des évènements extérieurs et des appels de la population. Les policiers en patrouille ont ainsi le temps de parler entre eux, notamment dans les voitures qui les isolent des interactions avec la population (Monjardet, 1996). Les échanges les plus importants concernent le cadrage de l'action: "S'il y a une bonne entente entre collègues, de toute façon, quand une intervention s'est mal passée, on va faire un remake, en reparler entre nous » (entretien, gardien de la paix). Cela peut aller des mots utilisés ou de l'attitude dans une interaction tendue avec un usager à des réflexions portant sur la répartition des tâches dans l'équipage, le positionnement lors d'une intervention par exemple. L'absence de ce genre de discussion se traduit par un plus grand nombre d'actions conflictuelles avec la population, de difficultés sur le terrain, comme le montrent nos observations. Ce cadrage est aussi une forme d'apprentissage du métier, les plus «anciens » (qui n'ont

privilégiés, les expressions significatives de la façon de caractériser les difficultés ou les plaintes ont été systématiquement notées. Ce travail n'est pas une analyse conversationnelle et seuls les entretiens ont été enregistrés (pour plus d'informations, cf. Loriol, Boussard et Caroly, 2004). 
parfois que quelques années de plus que les jeunes) faisant partager leur expérience. Il s'agit par exemple d'affiner le «regard» en expliquant comment «lire» le comportement d'usagers potentiellement suspects; de donner des informations sur la dangerosité de tel quartier ou de telle personne ; d'apprendre certaines ficelles du métier, etc. Le cadrage, enfin, peut être normatif, les discussions ayant pour effet de renforcer les règles et les valeurs du groupe, que ce soit dans les relations internes ou dans les interactions avec les usagers. Ainsi, un collègue qui, pour un problème de décompte des heures supplémentaires, est allé directement voir sa hiérarchie intermédiaire, sans passer d'abord par sa hiérarchie de proximité, est-il rabroué par ses collègues et «mis en quarantaine » (on ne lui adressera pratiquement plus la parole de toute la journée). De même, un policier à qui ses collègues reprochent de mettre systématiquement l'ensemble des amendes possibles aux usagers, même s'ils sont dociles, est traité de «verbalisator» car son comportement nuit à l'image de la police.

Comparativement à ce qui a été observé dans la police, les échanges informels sur le travail entre infirmières semblent moins nombreux et plus ponctuels. Cela est lié à la nature et à l'organisation du travail concerné. Passant de chambre en chambre pour faire leurs soins, les infirmières ont moins de temps de co-présence (Grosjean, Lacoste, 1999). L'intensification du travail du fait de la réduction des durées de séjour (les patients sont hospitalisés en phase aiguë, quand ils nécessitent davantage d'aide et de soins), la montée des tâches administratives, la raréfaction des temps de discussion ou de «tuilage» lors des changements d'équipe ou encore le recours croissant à des intérimaires, rendent les échanges plus difficiles (Estryn-Béhar, 2008).

La nature du travail effectué, généralement seul ou en binôme avec une aide-soignante, encourage peu le partage d'expérience. Agents de service, aides-soignantes, infirmières et médecins possèdent chacun leur propre vocabulaire, leurs propres conceptions du soin. Certains surveillants ou chefs de service ont tenté de mettre en place des réunions de service durant lesquelles sont discutés collectivement les problèmes, mais avec le risque de créer des conflits interpersonnels. Dans un contexte d'exercice individuel, porter un jugement public sur le travail d'une collègue risque d'être mal accepté, la norme étant que chacun évite de «trop s'occuper » du travail des autres. De plus, les conflits avec un supérieur ou un médecin sont plus souvent vécus sur 
Dossier : Pour une approche communicationnelle de l'individu au travail

le registre personnel par les infirmières ${ }^{11}$. Du coup, les échanges et l'entraide restent plus ponctuels et pratiques. Il s'agit par exemple de donner un conseil à une collègue sur la façon de se procurer tel instrument ou telle fourniture, de prévenir d'un changement de planning opératoire ou d'informer sur les habitudes d'un médecin. Les élèves infirmiers sont souvent envoyés auprès des patients jugés difficiles avec un minimum d'explications. Alors que pour les policiers (comme le montrent nos entretiens ou les travaux de Monjardet, 1986), l'école de police n'apporterait rien, toute la formation devant se faire sur le terrain sous la conduite de collègues expérimentés, les cadres infirmiers de proximité que nous avons interrogés estiment que la technique et la théorie ont été apprises en institut et qu'il ne manque que la pratique des soins aux nouveaux employés. Si les infirmiers parlent moins du travail, ils évoquent plus souvent le stress pour rendre compte de leurs difficultés.

\section{Maîtriser sy mboliquement l'environnement et d onner un sens à l'action}

Le cadrage du travail par les discussions entre collègues n'a pas seulement un aspect pratique, mais présente aussi une dimension symbolique en faisant vivre la culture professionnelle ${ }^{12} \mathrm{du}$ groupe, en donnant un sens acceptable aux contraintes du métier dès lors qu'elles sont perçues comme faisant partie des efforts normaux pour réaliser ce qui est défini collectivement comme le vrai travail, le «bel ouvrage».

Ainsi, les policiers, dans leurs discussions informelles réaffirment souvent leur hiérarchie des tâches, la valeur élevée attribuée à l'arrestation d'un délinquant violent et l'acceptation des risques pris pour y parvenir («chasse» en voiture, confrontation physique...). Lors d'une patrouille particulièrement calme, un gardien de la paix lance à la cantonade : "Ah, s'il avait un petit gars qui pouvait faire un vol dans une voiture, là, juste devant nous. Ou alors une agression sexuelle. Alors là je cours vite, je prends tous les risques pour l'attraper le gars, j'm'en fous, même s'il est armé ! ». Il s'agit de faire

\footnotetext{
11 À l'inverse des policiers pour qui ils seront plutôt pensés comme catégoriels (la base contre les gradés, la voie publique contre les bureaux, la tenue contre ceux en civil).

${ }^{12}$ À la suite des sociologues interactionnistes (Hughes, 1997 ; Strauss, 1992), la culture professionnelle n'est pas considérée comme un programme immuable intériorisé lors de la formation initiale, mais comme un ensemble d'attitudes sans cesse réactivées ou transformées au cours des confrontations entre collègues, avec les usagers, les autres groupes professionnels, etc.
} 
un travail qui a du sens par rapport aux représentations du rôle de la police : protéger le «faible» du «méchant». Mais le quotidien d'une patrouille de police-secours est plus terne et la «belle affaire» ne se présente qu'un petit nombre de fois au cours d'une vie professionnelle. Dès lors, les histoires que se racontent les policiers peuvent aider à combler le décalage entre le mythe et la réalité. Dans certaines brigades, les moments de gloire du commissariat (intervention sur un braquage, arrestation d'un détenu en cavale, éloignement d'un réseau de prostitution, etc.) sont régulièrement évoqués sous la forme d'anecdotes sans cesse enjolivées, comme l'a également observé un sociologue britannique (Holdaway, 1983). Dans certaines brigades, par des histoires édifiantes, les policiers, notamment les anciens, tentent de donner une dimension plus noble à des tâches routinières et peu prestigieuses. Ainsi l'importance du contrôle routier est-elle justifiée par des anecdotes à propos d'enfants blessés ou tués par des automobilistes imprudents. La «grandeur» de la verbalisation du stationnement illicite est évoquée à travers la défense des handicapés («Ils n'ont déjà pas grand chose dans la vie, alors si en plus on leur prend leur place!», gardien de la paix pendant une observation).

À l'hôpital, il n'existe pas de principe unique de valorisation du «beau travail». Suivant les services, la qualité de la prise en charge relationnelle ou la virtuosité technique seront plus ou moins mis en avant (Chauvenet, 1974). Des difficultés surviennent lorsque des divergences se font jour au sein d'une même équipe. Dans un service de chirurgie d'un hôpital de banlieue, les infirmières se répartissent en deux clans, aux origines sociales et professionnelles différentes (certaines étaient d'anciennes aides soignantes, originaires des DOMTOM). Communiquant peu, elles dénigrant le travail des autres, qualifiées soit de «piqueuses sans cœur», soit de «fainéantes passant leur temps à papoter ». Bien que partageant des lieux de sociabilité distincts, chaque groupe connaît les critiques des autres et en souffre dans son image professionnelle.

Dans le cas de la police comme pour les infirmières, la façon qu'ont les agents de caractériser les usagers vient renforcer le travail de construction du sens en délégitimant les demandes des usagers qui sortent du cadre de ce que les professionnels considèrent comme faisable ou en dehors de leur mission, mais aussi en valorisant celles qui renforcent l'idéal du métier. Les «mauvais malades», pour l'infirmière, sont ceux qui ne permettent pas de valoriser son rôle professionnel. C'est le cas tout d'abord pour celui qui n'est pas perçu comme un vrai malade; ceux que les étudiants en médecine étudiés 
Dossier : Pour une approche communicationnelle de l'individu au travail

par Howard Becker (2002) appelaient les «rosses ». Les patients dont les plaintes sont perçues comme imaginaires ou abusives sont dénigrés (par exemple en parlant de «syndrome méditerranéen ${ }^{13}$ ) et délaissés. Un vrai malade est aussi celui qui n'est pas jugé «responsable» de son état. Le toxicomane, l'alcoolique, le gros fumeur, l'obèse, celui qui est blessé suite à une bagarre est mal vu : il aurait pu «éviter» de se mettre dans cet état. Il peut être perçu comme moins «méritant» et les infirmières s'autorisent alors à moins donner d'elles-mêmes le concernant.

Pour les agents des brigades de police-secours, le «mauvais» délinquant est celui qui induit un travail peu valorisant. Les jeunes des cités, par exemple, apparaissent comme des «clients» ${ }^{14}$ peu intéressants. Non seulement les actes commis et le mérite qu'il y a à les interpeller sont de faible envergure; les policiers ont le sentiment qu'on leur fait jouer un rôle qui n'est pas le leur (pallier le manque éducatif des parents, jouer les assistantes sociales). À l'opposé de l'image du « grand bandit», ces jeunes de cité, sont qualifiés suivant les endroits de «crapauds», de «branleurs» et/ou de «petits merdeux». Ce sont les «mauvais» méchants parce qu'ils ne se comportent pas selon les règles du jeu établies par les policiers : ils manquent de respect, n'acceptent pas les sanctions, négocient les éléments de leur mise en cause, etc. De même, les plaignants trop exigeants ou dont les problèmes ne paraissent pas relever de l'action policière au sens strict (les «cas sociaux», les «victimes professionnelles ») sont décrits et traités avec une certaine distance ; leurs propos ne sont pas pris au sérieux.

À l'hôpital comme dans la police, ces «mauvais usagers» font l'objet, dans les discussions entre collègues, de médisances, de commérages, d'anecdotes présentées comme «typiques», qui permettent de disqualifier certaines demandes, de légitimer le fait de ne pas consacrer trop d'efforts et de temps à ce type de personnes avec lesquelles le «vrai travail» est impossible. Il s'agit donc d'un autre moyen de réduire l'écart entre l'idéal du métier et une réalité plus prosaïque, de faire exister une identité professionnelle particulière

\footnotetext{
${ }^{13}$ Exprimant différemment leur rapport à la maladie et à la douleur, les patients issus de l'immigration sont parfois mal compris et accusés d'exagérations.

${ }^{14}$ Pour les policiers que nous avons interrogés, leurs «clients» étaient les délinquants plutôt que les citoyens venant porter plainte, signe qu'ils ne se positionnent pas dans une logique de service aux usagers, mais dans une position régalienne (faire respecter l'ordre).
} 
Discussions informelles...

(Latimer, 2008) en marginalisant symboliquement et parfois réellement les demandes dissonantes.

\section{Une construction discursive du travail et du stress}

La comparaison entre policiers et infirmiers montre que, tant dans les discussions informelles spontanées que lors des entretiens, les premiers tendent à euphémiser la question du stress tandis que les seconds vont recourir plus fréquemment à ce terme ou à des entités proches (burn out, souffrance psychique, harcèlement, etc.). Il serait erroné d'expliquer cette différence en invoquant le machisme ou la valorisation de la virilité chez les policiers car c'est la conception même du stress qui est différente dans les deux milieux. Si les policiers parlent peu du stress, c'est que celui-ci est généralement vu comme le signe d'une double défaillance : celle de l'individu fragile qui n'a rien à faire sur la voie publique et celle du collectif (collègue et hiérarchie de proximité) qui n'a pas su régler à temps les problèmes et aider ceux qui étaient en difficulté. La solution «en interne» est toujours préférée et faire appel à une aide "extérieure » (psychologue, assistante sociale, haute hiérarchie) est estimé comme un manque de confiance. La solidarité et l'entraide sont considérées comme une norme, un devoir. À propos du suicide d'un collègue, une chef de brigade regrette de ne pas avoir su lui venir en aide en parlant avec lui de ses problèmes. Lors d'une observation, un autre chef de brigade explique: "Le stress, on l'a dans notre vie privée, en fait... heureusement qu'on se soutient entre nous, que le travail se passe bien. À la limite, on se ressource dans le travail, on oublie un peu nos problèmes...».

À l'inverse, parler de stress ou de burn out pour les infirmiers est souvent l'occasion d'évoquer la difficulté de leur métier et le nécessaire professionnalisme pour gérer les relations avec les malades. Le soutien des infirmiers entre eux est moins systématique. Du coup, le recours à des psychologues pour organiser et mettre en œuvre ce soutien psychologique est plus fréquent et mieux considéré que par les policiers.

Les différences entre métiers sont doublées par des différences entre services et brigades. "Il y a toujours des normes locales de communication qui instruisent elles-mêmes des normes locales d'activité : façons de dire et façon de faire se façonnent mutuellement, faisant émerger des cultures locales où ce qui se fait et se dit résulte d'un apprentissage » (Carcassonne, Glady, 2006). Ces cadrages locaux orientent également la mise en forme discursive des difficultés, leur gestion et le vécu du stress. Dans l'un des commissariats, les trois 


\section{Dossier : Pour une approche communicationnelle de l'individu au travail}

brigades de roulement sont typées. L'une d'entre elles présente un style décontracté avec de nombreuses plaisanteries, une forte solidarité et très peu de manifestations visibles de l'autorité du chef. Le travail semble toujours improvisé, décidé par le groupe à la dernière minute, mais les routines sont en fait bien rodées. L'humour constitue un moyen de gérer les problèmes ${ }^{15}$. Ainsi, la présence d'un sociologue travaillant sur le stress est l'occasion de se moquer des policiers réputés «stressés» dans d'autres services. De même, plaisanter à propos des femmes policières est une façon «gentille » mais ferme de les cantonner à un rôle particulier (soutien des victimes plus qu'usage de la force). Le stress est un sujet tabou ou ne concernant que les autres brigades ou commissariats. Une autre brigade présente des relations plus formelles et l'autorité du chef est fondée sur son expérience reconnue. Le chef n'impose pas les tâches, mais les activités sont légitimées par «l'évidence » ou une explication rationnelle. Parler du travail est une occasion de faire de la pédagogie et le stress doit être géré grâce au professionnalisme. Dans la troisième brigade, les relations sont plus tendues entre un chef autoritaire mais paternaliste et une partie de ses subordonnés. Les tâches imposées, dans un rapport de force parfois rude, sont plus souvent qu'ailleurs vécues comme des contraintes et il n'existe pas de consensus sur ce qu'est un «bon» travail policier. De fait, les conversations sur le travail sont rares et les plaintes (sur l'ambiance, les collègues, le chef, le travail) plus fréquentes qu'ailleurs. La plupart des brigades observées s'approchent plutôt du fonctionnement de la brigade «décontractée », sauf quand l'organisation du travail, le manque d'effectif et le turn-over entraînent un fonctionnement plus tendu sans possibilité de construire des représentations, des mythes et des valeurs communes.

Cette importance des conditions collectives et organisationnelles dans les formes de communication et la gestion des difficultés au travail est encore plus visible à l'hôpital. Par exemple, dans les premiers services de soins palliatifs (Castra, 2003) un recrutement de soignants motivés et expérimentés, des moyens humains et matériels importants, une sélection écartant les malades déments ou les pathologies

${ }^{15}$ Il est révélateur que les plaisanteries, parmi les policiers soient plus tournées vers les collègues comme moyens de réguler les problèmes relationnels internes tandis que chez les infirmiers l'humour est plutôt dirigé vers les malades ou leur famille; l'humour noir et les blagues de «salle de garde» pouvant soulager les tensions liées à cette relation perçue comme potentiellement stressante. 
imprévisibles, a rendu possible de nombreuses discussions formelles et informelles sur le travail, l'élaboration et la mise en œuvre au moins partielle d'un «accompagnement à la bonne mort» (réduction des souffrances physiques et morales du patient, «acceptation apaisée » de sa mort). À l'inverse, dans nombre de services de médecine de petits hôpitaux locaux, où meurent souvent des patients âgés déments atteints de polypathologies et où les soins de base sont à peine assurés faute de personnel, il est plus difficile de parler «d'accompagnement à la bonne mort» et les conversations sur le travail sont moins fréquentes du fait des conflits sur la définition de ce qu'est un «bon travail ».

\section{Conclusion}

Ce dernier exemple montre que la construction collective des conditions de travail ne peut être uniquement discursive car il faut que les mythes, l'idéal, les valeurs, promus en parole s'accompagnent d'agencements organisationnels (Girin, 2001) qui les rendent - au moins partiellement - possibles. La capacité à construire et à faire exister une vision positive du travail permet la médiatisation des difficultés qui ne se traduisent pas automatiquement en plaintes. L'interruption des tâches, des consignes floues ou mal définies, de fortes demandes du public, de lourdes responsabilités, etc. sont d'autant mieux supportées que le collectif de travail s'est doté de représentations fortes et partagées du travail et de ses limites. En échangeant avec leurs collègues, les salariés produisent collectivement des représentations des valeurs et une identité professionnelle (Alter, 2009) qui, pour être viables doivent se traduire, au moins en partie, dans l'organisation et les moyens disponibles créant ainsi des effets de boucles bien mis en évidence par les travaux constructivistes (Hacking, 2001) : les représentations orientent les perceptions et les choix des acteurs et finissent par modifier l'environnement de façon à ce que celui-ci confirme, en retour, les représentations initiales.

\section{Bibliographie}

ALTER N. Donner et prendre. La coopération en entreprise. Paris : La découverte, 2009, $231 \mathrm{p}$.

BATESON G. Vers une écologie de l'esprit. Tome 1. Paris : Le Seuil. 1977, $285 \mathrm{p}$.

BECKER H. Les ficelles du métier. Paris: La découverte, 2002, $354 \mathrm{p}$. 
Dossier : Pour une approche communicationnelle de l'individu au travail

CARCASSONNE M., GLADY M. Langage, travail et organisation, in: ALTER N. Sociologie du monde du travail. Paris, PUF. 2006, pp. 209-223.

CASTRA M. Bien mourir. Sociologie des soins palliatifs. Paris : PUF. 2003, $365 \mathrm{p}$.

CHAUVENET A. L'infirmière hospitalière technicienne ou travailleur social. in : Projet. 1974, $n^{\circ}$ 90, pp. 1135-1148.

COBB S. Social Support as a Moderator of Life Stress. in : Psychosomatic Medecine. 1976, Vol. 38, pp. 300-314.

FOSTER E., BOCHNER A. Social constuctionnist perspective in communication Research. in : HOLSTEIN J., GUBRIUM J. Handbook of constructionist research. Londres: Guilford, 2008, pp. 85-106.

ESTRYN-BEHAR M. Santé et satisfaction des soignants au travail en France et en Europe. Rennes : Presses de l'EHESP, 2008, 383 p.

FREUD S. Cinq leçons sur la psychanalyse, Paris: Payot, 2004 (1909), 208 p.

GIRIN J. La théorie des organisations et la question du langage. in : BORZEIX A., FRAENKEL B. Langage et travail. Paris : CNRS éditions, 2001, pp. 167-184.

GOFFMAN E. Les Cadres de l'expérience. Paris: Minuit, 1991, $573 \mathrm{p}$.

GROSJEAN M., LACOSTE M. Communication et intelligence collective. Le travail à l'hôpital. Paris : PUF, 1999, 225 p.

HACKING I. Entre science et réalité, La construction sociale de quoi ? Paris : La découverte, 2001, 240p. (Textes à l'appui)

HANIQUE F. Le sens du travail. Chronique de la modernisation au guichet. Ramonville : ERES, 2004, 300 p.

HOLDAWAY S. Inside the British police. A force at work. Oxford: Basil Blackwell, 1983, 196 p.

HUGHES E. Le regard sociologique. Essais choisis. Paris: Editions de l'EHESS, 1997, $344 \mathrm{p}$.

LATIMER J. Critical constructionism in nursing research. in : HOLSTEIN J., GUBRIUM J. Handbook of constructionist research. Londres: Guilford, 2008, pp. 153-170. 
Discussions informelles...

LORIOL M., BOUSSARD V., CAROLY S. Constructions du stress, psychologisation du social et rapport au public. in: Rapport pour l'ACI Travail dans la fonction publique. Paris : Laboratoire Georges Friedmann, 2004, 172 p.

MILBURN P. La compétence relationnelle : maîtrise de l'interaction et légitimité professionnelle, Avocats et médiateurs. in: Revue française de sociologie, 2002, 43, 1, pp. 47-72.

MONJARDET D. Ce que fait la police. Sociologie de la force publique. Paris : La Découverte, 1996, 316 p.

PAUGAM S. Le salarié de la précarité. Les nouvelles formes de l'intégration professionnelle. Paris : PUF, 2000, 437 p.

RIME B. Le partage social des émotions. Paris : PUF, 2005, 420 p.

VIGOUR C. La comparaison dans les sciences sociales : Pratiques et méthodes. Paris, La Découverte, 2004, 336 p.

STRAUSS A. La trame de la négociation. Sociologie qualitative et interactionnisme. Paris : L'Harmattan, 1992, 311 p. 